\title{
Heavy-light mesons beyond the ladder approximation
}

\author{
Pianpian Qin ${ }^{1}{ }^{1}$ Si-xue Qin, ${ }^{2, *}$ and Yu-xin Liu ${ }^{1,3,4, \dagger}$ \\ ${ }^{1}$ Department of Physics and State Key Laboratory of Nuclear Physics and Technology, Peking University, \\ Beijing 100871, China \\ ${ }^{2}$ Department of Physics, Chongqing University, Chongqing 401331, China \\ ${ }^{3}$ Collaborative Innovation Center of Quantum Matter, Beijing 100871, China \\ ${ }^{4}$ Center for High Energy Physics, Peking University, Beijing 100871, China
}

(Received 20 December 2019; accepted 6 April 2020; published 16 June 2020)

\begin{abstract}
The heavy-light mesons are studied within the framework of Dyson-Schwinger equations of QCD. Inspired by the axial-vector Ward-Takahashi identity resulting from the chiral symmetry, we propose a truncation scheme beyond the ladder approximation without introducing any additional parameters. For the pseudoscalar and vector heavy-light mesons, the obtained mass spectrum has the level of relative errors at $5 \%$ compared with experimental data and lattice-QCD results. For the leptonic decay constants, our results are comparable with those from experiments and/or lattice QCD. For some channels, the discrepancies are sizable but significantly smaller than those using the equal spacing rule. The truncation scheme proposed in this work is simple and could be improved and applied to study other open flavor hadrons including both mesons and baryons.
\end{abstract}

DOI: 10.1103/PhysRevD.101.114014

\section{INTRODUCTION}

In recent decades, many heavy-light mesons are observed in experiments [1], which has attracted a lot of interest. The study of heavy-light mesons provides a way to understand dynamical chiral symmetry breaking (DCSB) [2-4]-one of the most fascinating features of the quantum chromodynamics (QCD). Nevertheless it is still very challenging. The heavy-light mesons are two-body bound states of heavy and light (anti)quarks, e.g., $c \bar{d}, c \bar{u}, c \bar{s}$, etc., which are highly flavor unsymmetric. Thus, the successful descriptions require a systematic and self-consistent understanding of strong interaction in both ultraviolet and infrared regions. Herein, nonperturbative approach of QCD is the key for solving the problem.

Several nonperturbative approaches of QCD have been developed, such as the lattice QCD (1QCD) [5-8], the Dyson-Schwinger equations (DSEs) [9-15], the functional renormalization group (FRG) [16-18], and so on. Within the DSE framework, mesons are described with the twobody Bethe-Salpeter equation (BSE) $[9,11,12,19-23]$. As input, the quark propagator must be solved by the one-body

\footnotetext{
sqin@cqu.edu.cn

yxliu@pku.edu.cn
}

Published by the American Physical Society under the terms of the Creative Commons Attribution 4.0 International license. Further distribution of this work must maintain attribution to the author(s) and the published article's title, journal citation, and DOI. Funded by SCOAP. gap equation. The key point is that the one-body and the two-body equations must be constructed in a self-consistent way to preserve QCD's symmetries. For instance, the chiral symmetry is crucial to realize pion's twofold role as quark-antiquark bound state and Nambu-Goldstone boson [21-23].

The simplest symmetry-preserving scheme is the socalled rainbow-ladder (RL) truncation [9,22-30], which approximates the dressed quark-gluon interaction vertex as the bare one and the quark-antiquark scattering kernel as the one-gluon exchange form. Since the bare vertex is flavor-blind and the one-gluon exchange lacks of spin-orbit repulsion, the RL truncation can only work for ground state pseudoscalar and vector mesons with symmetric flavors, e.g., the light-light mesons [23-26,29] and the heavy-heavy mesons [31-37].

For heavy-light mesons, a sophisticated improvement of the RL truncation must be developed. Recently, following this direction, some progress has been made [38-47]. In this work, we implement the DSE approach to study properties of heavy-light mesons. Inspired by the axialvector Ward Takahashi identity (AV-WTI) [23,25], we propose a flavor-sensitive Bethe-Salpeter kernel beyond the simplest ladder approximation. The interaction kernel degenerates to the ladder approximation for flavorsymmetric systems, and thus can be considered as a generalization of the latter. Without introducing any new parameter, the mass spectra and leptonic decay constants of heavy-light mesons in pseudoscalar and vector channels are well reproduced, self-consistently and systematically. 
The remainder of this article is organized as follows. In Sec. II, we describe the DSE approach and the setups. In Sec. III, we fit the parameters with the light-light and heavy-heavy systems first, and then present results of heavy-light mesons. The comparisons with experimental data and results of lattice QCD are also included. Section IV provides a summary and some brief remarks.

\section{THEORETICAL FRAMEWORK}

\section{A. The gap equation}

The quark gap equation reads [9]

$$
S^{-1}(p)=Z_{2}\left(\not \not p p+Z_{m} m\right)+\Sigma(p),
$$

with the self-energy

$\Sigma(p)=g^{2} Z_{1} \int_{q}^{\Lambda} D_{\mu \nu}(p-q) \frac{\lambda^{a}}{2} \gamma_{\mu} S(q) \frac{\lambda^{a}}{2} \Gamma_{\nu}(q, p)$,

where $Z_{1}, Z_{2}$, and $Z_{m}$ are the vertex, quark wave function, and mass renormalization constants, respectively; $m$ is the renormalized current quark mass; $\lambda^{a}$ are the color matrices; $\int_{q}^{\Lambda}$ represents a Poincáre invariant regularization of the four-dimensional integral, with $\Lambda$ the ultraviolet regularization mass scale; $\Gamma_{\nu}$ and $D_{\mu \nu}$ are the dressed quark-gluon vertex and the dressed gluon propagator, respectively. A schematic demonstration of the equation is illustrated in Fig. 1.

The solution of the gap equation, i.e., the dressed quark propagator, can usually be decomposed as

$$
\begin{gathered}
S(p)=-i \not p \sigma_{v}\left(p^{2}\right)+\sigma_{s}\left(p^{2}\right), \\
S^{-1}(p)=i \not p A\left(p^{2}\right)+B\left(p^{2}\right),
\end{gathered}
$$

with the momentum subtraction renormalization condition

$$
\left.S^{-1}(p)\right|_{p^{2}=\zeta^{2}}=i \not \varnothing+m^{\zeta}
$$

where $\zeta$ is the renormalization point and $m^{\zeta}$ the renormalized current-quark mass, the dressed quark mass function reads

$$
M\left(p^{2}\right)=B\left(p^{2}, \zeta^{2}\right) / A\left(p^{2}, \zeta^{2}\right)
$$

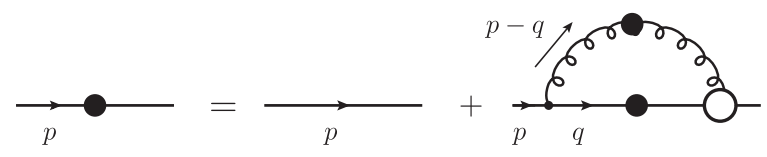

FIG. 1. The Feynmann diagram for the Dyson-Schwinger equation of the quark propagator in Eq. (1). Solid line with black blob: dressed quark propagator; solid curve with black blob: dressed gluon propagator; white circle: dressed quark-gluon interaction vertex; black dot: bare quark-gluon interaction vertex. which is independent of the renormalization point $\zeta$. Note that $m^{\zeta}$ is the mass function evaluated at the renormalization point $m^{\zeta}=M\left(\zeta^{2}\right)$.

To solve the gap equation, the dressed quark-gluon vertex and the dressed gluon propagator must be specified. The rainbow part of the RL approximation can be expressed as $(k=p-q$, as shown in Fig. 1)

$$
Z_{1} g^{2} D_{\mu \nu}(k) \Gamma_{\nu}(q, p)=k^{2} \mathcal{G}\left(k^{2}\right) D_{\mu \nu}^{\mathrm{free}}(k) \gamma_{\nu},
$$

where $D_{\mu \nu}^{\mathrm{free}}(k)=\left(\delta_{\mu \nu}-\frac{k_{\mu} k_{\nu}}{k^{2}}\right) \frac{1}{k^{2}}$ is the Landau-gauge free gluon propagator. The interaction model $\mathcal{G}\left(k^{2}\right)$ can be written as

$$
k^{2} \mathcal{G}\left(k^{2}\right)=k^{2} \mathcal{G}_{\mathrm{IR}}\left(k^{2}\right)+4 \pi \tilde{\alpha}_{p \mathrm{QCD}}\left(k^{2}\right),
$$

where $\tilde{\alpha}_{p \mathrm{QCD}}\left(k^{2}\right)$ is a bounded and monotonically decreasing regular continuation of the perturbative QCD running coupling to all values of spacelike- $k^{2}$. The $\mathcal{G}_{\mathrm{IR}}\left(k^{2}\right)$ is a model for the effective interaction in infrared region and dominates in the region $|k|<\Lambda_{\mathrm{QCD}}$. The form of $\mathcal{G}_{\mathrm{IR}}\left(k^{2}\right)$ determines whether the DCSB and/or confinement can be realized. Up to now, several ansatze to represent the effective interaction in infrared region have been established. The commonly used ones include the MunczekNemirovsky model [48], the Roberts-Williams model [9,25], the Frank-Roberts model [24], the Maris-Tandy model [26] and the Qin-Chang-Liu-Roberts-Wilson (QCLRW) model [49].

With the QCLRW model [49], the interaction is expressed as $\left(s=k^{2}\right)$

$$
\mathcal{G}(s)=\frac{8 \pi^{2}}{\omega^{4}} D e^{-s / \omega^{2}}+\frac{8 \pi^{2} \gamma_{m} \mathcal{F}(s)}{\ln \left[\tau+\left(1+s / \Lambda_{\mathrm{QCD}}^{2}\right)^{2}\right]},
$$

where $\mathcal{F}(s)=\left(1-e^{-s / 4 m_{t}^{2}}\right) / s$ with $m_{t}=0.5 \mathrm{GeV} ; \gamma_{m}=$ $12 /\left(33-2 N_{f}\right)$ is the dimension anomaly with the flavor number $N_{f} ; \tau=e^{2}-1$ is a constant. Following Ref. [50], we take $N_{f}=5$ and $\Lambda_{\mathrm{QCD}}=0.36 \mathrm{GeV}$.

As the same as that in Refs. [9,26], the second term of Eq. (9) is the Coulomb interaction originating from the one-gluon-exchange force. While, different from that in Ref. $[9,26]$, the first term gives an infrared-saturated interaction which incorporates the modern results of the gluon running mass and the running coupling strength [51-56], and is consistent with that given in general analysis [57].

The characteristic of Eq. (9) can also be parametrized as $[58,59]$

$$
\mathcal{G}\left(k^{2}\right)=\frac{4 \pi \alpha_{R L}\left(k^{2}\right)}{k^{2}+m_{g}^{2}\left(k^{2}\right)}, \quad m_{g}^{2}\left(k^{2}\right)=\frac{M_{g}^{4}}{k^{2}+M_{g}^{2}},
$$


where $m_{g}\left(k^{2}\right)$ is the dynamical mass of the gluon and $M_{g}$ is a mass scale. $\alpha_{R L}\left(k^{2}\right)$ is the effective running-coupling strength, which is strong in the infrared and has the oneloop perturbative feature in the ultraviolet. Therefore, the interaction achieves its maximum value at the deep infrared $k^{2}=0$. The running behavior and the gluon mass scale are consistent with not only the early conjecture [60] but also the modern DSE and lattice-QCD results [51-56]. And the details about the consistency between a gluon mass and gauge invariance can be found in Ref. [61].

Many studies (e.g., Refs. [29,30,37,46,50,53,62-70]) have shown that the above mentioned interaction model is quite successful. The parameters $D$ and $\omega$ control the strength and the width of the interaction, respectively, and in turn, dominate the emergence of the DCSB [71,72]. In fact, properties of light-quark ground-state vector- and isospin-nonzero pseudoscalar mesons are insensitive to variations of $\omega \in[0.4,0.6] \mathrm{GeV}$, as long as

$$
\varsigma^{3}:=D \omega=\text { constant. }
$$

Since the interaction model absorbs the dressed effects of the quark-gluon interaction vertex and the gluon propagator, for light quark systems, it has much larger strength compared with that of the realistic gauge-sector interaction at infrared momenta [49] and the one determined by solving the coupled equations of the quark propagator and the quark-gluon interaction vertex [56]. However, straightforward analysis [27] and numerical calculation [56] show that the infrared boost to the RL truncation almost vanishes in the heavy-quark limit. Then, it is expected that the RL truncation provides a reasonable approximation for the systems involving only heavy quarks so long as one employs a smaller strength. This will be discussed in detail in Sec. III.

\section{B. The Bethe-Salpeter equation}

In terms of Green functions, properties of mesons are encoded in the quark-antiquark scattering matrices. The corresponding bound-state equation for mesons is the homogenous Bethe-Salpeter equation, which is a matrixequation in the spinor space and reads

$$
\Gamma(k ; P)=\int_{q}^{\Lambda} S_{f}\left(q_{+}\right) \Gamma(q ; P) S_{g}\left(q_{-}\right) K(k, q ; P),
$$

where $P^{2}=-M^{2}$ with the meson mass $M ; q_{+}=q+\alpha P$ and $q_{-}=q-(1-\alpha) P$ with the momentum fraction $\alpha \in[0,1] ; f$ and $g$ denote the quark and antiquark flavors; $\Gamma(k, P)$ is the Bethe-Salpeter amplitude (BSA); $K(k, q ; P)$ is the quark-antiquark scattering kernel. The dressed quark propagators are fed with the solution of the gap equation. A schematic view is displayed in Fig. 2.

According to the $J^{P}$ quantum numbers, the BSA of the pseudoscalar $\left(J^{P}=0^{-}\right)$and vector $\left(J^{P}=1^{-}\right)$mesons are

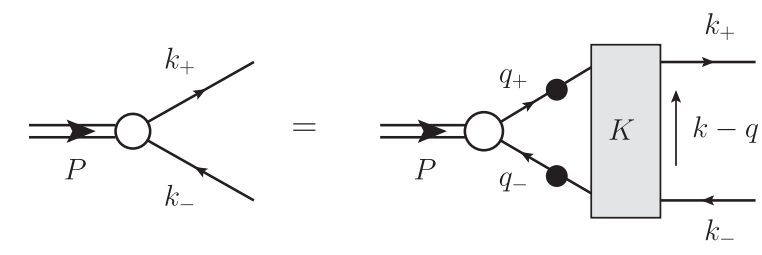

FIG. 2. The Feynmann diagram for the homogenous BetheSalpeter equation in Eq. (12). White circle: Bethe-Salpeter amplitude; solid line with black blob: dressed quark propagator; shaded rectangular: quark-quark interaction kernel.

matrices in the 4-dimensional spinor space and can be expanded with the corresponding set of basis $[73,74]$ :

$$
\begin{aligned}
& \Gamma_{0^{-}}(k ; P)=\sum_{i=1}^{4} \tau_{0^{-}}^{i}(k ; P) \mathcal{F}_{i}\left(k^{2}, z_{k} ; P^{2}\right), \\
& \Gamma_{1^{-}}(k ; P)=\sum_{i=1}^{8} \tau_{1^{-}}^{i}(k ; P) \mathcal{F}_{i}\left(k^{2}, z_{k} ; P^{2}\right) .
\end{aligned}
$$

Concerning the full Lorentz structure of the four-body scattering kernel and the dressed effects of interactions, the basis of the pseudoscalar channel is written as $[25,29,73-75]$

$$
\begin{array}{ll}
\tau_{0^{-}}^{1}=i \gamma_{5}, & \tau_{0^{-}}^{2}=\gamma_{5} \not P, \\
\tau_{0^{-}}^{3}=\gamma_{5} \not k(k \cdot P), & \tau_{0^{-}}^{4}=i \gamma_{5} \sigma^{P, k} .
\end{array}
$$

And the basis of the vector channel reads [29,73-75]

$$
\begin{array}{llrl}
\tau_{1^{-}}^{1} & =i \gamma_{\mu}^{T}, & \tau_{1^{-}}^{2} & =i k_{\mu}^{T} \not k, \\
\tau_{1^{-}}^{3} & =i k_{\mu}^{T} \not p(k \cdot P), & & \tau_{1^{-}}^{4}=\gamma_{5} \epsilon_{\mu \nu \alpha \beta}^{T} \gamma_{\nu} k_{\alpha} P_{\beta}, \\
\tau_{1^{-}}^{5} & =k_{\mu}^{T}, & & \tau_{1^{-}}^{6}=\sigma_{\mu \nu}^{T} k_{\nu}(k \cdot P), \\
\tau_{1^{-}}^{7} & =\sigma_{\mu \nu}^{T} P_{\nu}, & & \tau_{1^{-}}^{8}=k_{\mu}^{T} \sigma_{\alpha \beta}^{T} k_{\alpha} P_{\beta},
\end{array}
$$

where $l_{\mu}^{T}=P_{\mu \nu} l_{\nu}$ with $P_{\mu \nu}=\delta_{\mu \nu}-\frac{P_{\mu} P_{\nu}}{P^{2}}$ as the transverse projector on the meson momentum $P$.

The coefficient functions $\mathcal{F}_{i}\left(k^{2}, z_{k} ; P^{2}\right)$ can be expanded in terms of the Chebyshev polynomials [74] as

$$
\mathcal{F}^{J P}\left(k^{2}, k \cdot P\right)=\sum_{j=0}^{\infty} a_{j}\left(k^{2}\right) U_{j}\left(k \cdot P / \sqrt{k^{2} P^{2}}\right),
$$

where $U_{j}\left(k \cdot P / \sqrt{k^{2} P^{2}}\right)$ are the Chebyshev polynomials of the second kind and the $a_{j}\left(k^{2}\right)$ are the corresponding Chebyshev moments.

The space inversion $(P)$ and charge conjugation $(C)$ transformations for the BSA are defined as

$$
\Gamma(k ; P) \stackrel{P}{\rightarrow} \hat{P} \Gamma(\tilde{k} ; \tilde{P}) \hat{P}^{-1},
$$




$$
\Gamma(k ; P) \stackrel{C}{\rightarrow} \bar{\Gamma}(k ; P)=\hat{C} \Gamma^{t}(-k ; P) \hat{C}^{-1},
$$

with $\tilde{k}=\left(k_{4},-\vec{k}\right), \hat{P}=\gamma_{4}$, and $\hat{C}=\gamma_{2} \gamma_{4}$. The basis are chosen to have specific $P$ - and $C$-parity.

To normalize the BSA, we apply the Nakanish normalization condition $[76,77]$

$$
\left(\frac{\partial \ln (\lambda)}{\partial P^{2}}\right)^{-1}=\operatorname{tr} \int_{k}^{\Lambda} \bar{\Gamma}(k ;-P) S\left(k_{+}\right) \Gamma(k ; P) S\left(k_{-}\right),
$$

where $\bar{\Gamma}(k ;-P)$ is the charge conjugation of $\Gamma(k ;-P)$ as defined in Eq. (19). With the normalized BSAs, the leptonic decay constants of pseudoscalar and vector mesons are defined as

$$
\begin{array}{r}
f_{0^{-}} P_{\mu}=\frac{Z_{2}}{\sqrt{2}} \operatorname{tr} \int i \gamma_{5} \gamma_{\mu} S_{f}\left(k_{+}\right) \Gamma_{0^{-}}(k, P) S_{g}\left(k_{-}\right), \\
f_{1^{-}} M=\frac{Z_{2}}{3 \sqrt{2}} \operatorname{tr} \int \gamma_{\mu} S_{f}\left(k_{+}\right) \Gamma_{1^{-}}^{\mu}(k, P) S_{g}\left(k_{-}\right) .
\end{array}
$$

\section{The scattering kernel}

Now the scattering kernel is the only unknown piece of the whole issue. To construct it, we recall the axial-vector Ward-Takahashi identity (AV-WTI) [23,25] derived from the QCD chiral transformation, which reads

$$
\begin{gathered}
P_{\mu} \Gamma_{5 \mu}^{f g}(k ; P)+i\left(m_{f}+m_{g}\right) \Gamma_{5}^{f g}(k ; P) \\
=S_{f}^{-1}\left(k_{+}\right) i \gamma_{5}+i \gamma_{5} S_{g}^{-1}\left(k_{-}\right),
\end{gathered}
$$

where $\Gamma_{5 \mu}^{f g}(k ; P)$ and $\Gamma_{5}^{f g}(k ; P)$ are the axial-vector and pseudoscalar vertex, respectively, with two quark flavors denoted by $f$ and $g$. Note that the identity is modelindependent and scheme-independent. The vertices can be determined by the inhomogeneous Bethe-Salpeter equation

$$
\begin{aligned}
& \Gamma_{5 \mu}^{f g}(k ; P)=\gamma_{\mathrm{av}}+\int_{q} K(q, k ; P) S_{f}\left(q_{+}\right) \Gamma_{5 \mu}^{f g}(q ; P) S_{g}\left(q_{-}\right), \\
& \Gamma_{5}^{f g}(k ; P)=\gamma_{\mathrm{ps}}+\int_{q} K(q, k ; P) S_{f}\left(q_{+}\right) \Gamma_{5}^{f g}(q ; P) S_{g}\left(q_{-}\right),
\end{aligned}
$$

where the inhomogeneous terms $\gamma_{\mathrm{av}}=Z_{2} \gamma_{5} \gamma_{\mu}$ and $\gamma_{\mathrm{ps}}=$ $Z_{2} Z_{m} \gamma_{5}$. The poles of the vertices correspond to the array of meson states. More specifically, the pole location gives the meson mass, and the pole residue relates to the corresponding wave function.

In the chiral limit, the current mass term vanishes and the pion must be a massless Goldstone boson. In the neighborhood $P^{2} \simeq 0$, we have

$$
\Gamma_{5 \mu}(k ; P) \sim \frac{2 f_{\pi} \Gamma_{\pi}(k ; P) P_{\mu}}{P^{2}}+\text { regular term }
$$

where $\Gamma_{\pi}(k ; P)$ is the pion BSA, i.e.,

$$
\begin{aligned}
\Gamma_{\pi}(k ; P)= & \gamma_{5}\left[i E_{\pi}(k ; P)+\not P F_{\pi}(k ; P)\right. \\
& \left.+\not k(k \cdot P) G_{\pi}(k ; P)+\sigma_{\mu \nu} k_{\mu} P_{\nu} H_{\pi}(k ; P)\right],
\end{aligned}
$$

Using the AV-WTI, one can obtain [78]

$$
f_{\pi} E_{\pi}(k ; 0)=B\left(k^{2}\right),
$$

where $B\left(k^{2}\right)$ is the scalar function defined in Eq. (4). It is apparent that the two-body problem is related to the onebody one and reveals that the pion can exist if and only if the chiral symmetry is dynamically broken.

The kernel with the one-gluon exchange form, which is called the "ladder" approximation $[9,21,22]$, can be expressed as $(l=k-q)$

$K_{t u}^{r s}(q, k ; P)=-\mathcal{G}\left(l^{2}\right) l^{2} D_{\mu \nu}^{\mathrm{free}}(l)\left(\gamma_{\mu} \frac{\lambda^{a}}{2}\right)_{\mathrm{tr}}\left(\gamma_{\nu} \frac{\lambda^{a}}{2}\right)_{s u}$,

where $t, u, r, s$ denote the Dirac and color indices. With the RL approximation, i.e., Eqs. (7) and (26), the solved quark propagator and vertices satisfy the identity in Eq. (23). In other words, the RL approximation is a symmetry-preserving truncation scheme.

In order to construct an interaction kernel beyond the ladder approximation, we first insert the gap equation and the inhomogeneous BSE into the AV-WTI. It is found that the inhomogeneous terms of BSE are canceled by the bare terms of the gap equation. In other word, the AV-WTI is satisfied in the tree level. Then, we obtain the identity of the interaction terms (for some details of the derivation, please refer to the Appendix of this paper) as

$$
\begin{aligned}
\int_{q} K(q, k ; P)\left[S_{f}\left(q_{+}\right) \gamma_{5}+\gamma_{5} S_{g}\left(q_{-}\right)\right] \\
\quad=-\int_{q} \gamma_{\mu}\left[D_{\mu \nu}^{f}(l) S_{f}\left(q_{+}\right) \gamma_{5}+D_{\mu \nu}^{g}(l) \gamma_{5} S_{g}\left(q_{-}\right)\right] \gamma_{\nu},
\end{aligned}
$$

where the rainbow approximation has been implemented and all the indices are suppressed for simplicity. Note that the square bracket on the left-hand side of the above equation comes from reusing the AV-WTI for the vertices, i.e., $\Gamma_{5 \mu}^{f g}(q, P)$ and $\Gamma_{5}^{f g}(q, P)$. Since the quark propagators with different flavors may have different effective interaction strengths [56], we take then $D_{\mu \nu}^{f}, D_{\mu \nu}^{g}$ to specify each of them for clarity.

After some derivations inspired by Eq. (27) (for some details about the derivation, please refer to the Appendix), we propose the kernel as (the color matrices are suppressed) 

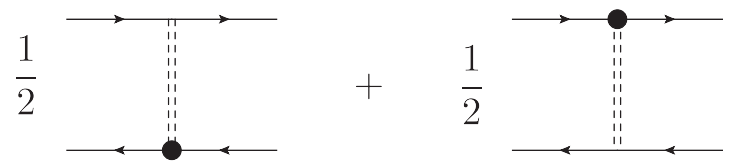

FIG. 3. The Feynmann diagram for the kernel in Eq. (28). The double-dashed lines denote the averaged interaction. The black blobs denote the elements in Eq. (29), which contains fully dressed effects. Note that the black blobs contain dressed effects and involve three independent momenta, i.e., $l$ and $q_{ \pm}$, which is not simply a three-point object. It is very hard to expand the kernel by the regular Feynman diagrams.

$$
K=-\frac{1}{2} \gamma_{\mu} \otimes \Sigma \cdot D_{\mu \nu}^{\Sigma} \cdot \gamma_{\nu}-\frac{1}{2} \gamma_{\nu} \cdot D_{\mu \nu}^{\Sigma} \cdot \Sigma \otimes \gamma_{\mu}
$$

with

$$
\begin{aligned}
D_{\mu \nu}^{\Sigma} & =\left[D_{\mu \nu}^{f}(l) S_{f}\left(q_{+}\right)+D_{\mu \nu}^{g}(l) S_{g}\left(q_{-}\right)\right] \gamma_{5}, \\
\Sigma & =\gamma_{5}\left[S_{f}\left(q_{+}\right)+S_{g}\left(q_{-}\right)\right]^{-1} .
\end{aligned}
$$

The kernel can be illustrated by the Feynmann diagram shown in Fig. 3. If the two quarks have the same flavor, i.e., $D^{f}=D^{g}$, the kernel degenerates into Eq. (26) and thus can be considered as a parameter-free generalization of the ladder approximation.

Straightforward analysis suggests that the kernel actually expresses an average of the effective interactions of the two flavors. For example, in the ultraviolet limit, i.e., $q \rightarrow \infty$, we obtain

$$
K \rightarrow-\gamma \otimes \gamma\left(\frac{D^{f}+D^{g}}{2}\right)
$$

which is obviously a simple average of $D^{f}$ and $D^{g}$. As another example, in the infrared limit, i.e., $q \rightarrow 0$, we have the kernel for $P \sim 0$ as

$$
K \rightarrow-\gamma \otimes \gamma\left(\frac{D^{f} \sigma_{s}^{f}(0)+D^{g} \sigma_{s}^{g}(0)}{\sigma_{s}^{f}(0)+\sigma_{s}^{g}(0)}\right),
$$

which is also an average of $D^{f}$ and $D^{g}$ but weighted by the corresponding quark dressing functions.

\section{CALCULATIONS AND RESULTS}

Recalling our scheme, one can know easily that, to carry out the calculations for heavy-light mesons, one needs to calibrate the parameters $D$ and $\omega$ (or equivalently, $\omega$ and $\varsigma$ ) in the gluon propagator and the current quark mass $m$ in the gap equation of light quark and heavy quark. We carry out the calibration with light flavor mesons and heavy flavor mesons as the follows.

\section{A. Calibration of the parameters}

\section{Light quark sector}

Following Ref. [50], for the mesons consisting of light quarks $u, d, s$, we take the interaction parameters as

$$
\omega_{q}=0.5 \mathrm{GeV}, \quad \varsigma_{q}=0.8 \mathrm{GeV} .
$$

In the isospin symmetric limit: $m_{u}=m_{d}$, fitting with the underlined data listed in Table I, we take

$$
m_{u / d}^{\zeta_{19}}=3.3 \mathrm{MeV}, \quad m_{s}^{\zeta_{19}}=74.6 \mathrm{MeV},
$$

with which the masses and leptonic decay constants of light mesons are computed and listed in Tables I and II. The renormalization-group-invariant masses are $\hat{m}_{u / d}=$ $6.3 \mathrm{MeV}, \hat{m}_{s}=146 \mathrm{MeV}$. The one-loop-evolved masses at $2 \mathrm{GeV}$ are $m_{u / d}^{2 \mathrm{GeV}}=4.8 \mathrm{MeV}, m_{s}^{2} \mathrm{GeV}=110 \mathrm{MeV}$. The Euclidean constant quark masses are obtained

$$
M_{u / d}^{E}=0.41 \mathrm{GeV}, \quad M_{s}^{E}=0.57 \mathrm{GeV},
$$

defined as $M_{q}^{E}=\left\{k \mid M_{q}\left(k^{2}\right)=k\right\}$, where $M_{q}\left(k^{2}\right)$ is the quark mass function.

\section{Heavy quark sector}

For the heavy quark mesons, we follow the guide aforementioned and take the interaction parameters as

$$
\omega_{Q}=0.8 \mathrm{GeV}, \quad \varsigma_{Q}=0.6 \mathrm{GeV},
$$

which shape a much weaker effective interaction compared with that for the light quark mesons.

With the heavy quark current masses (fitted with the underlined data in Table I)

TABLE I. Masses of pseudoscalar mesons calculated herein compared with those from the $\mathrm{QCD}$ and experimental data. The ESR results are also presented for comparison. The 1 QCD results are taken from: $M_{B_{c}}-$ Ref. [79]; $M_{D}$ and $M_{D_{s}^{ \pm}}-$Ref. [80]; $M_{B}$ and $M_{B_{s}^{ \pm}}-$Ref. [81]. The experimental data (labeled as Expt.) are taken from Ref. [1]. The underlined values are fitted by the experimental data.

\begin{tabular}{lcccc}
\hline \hline Meson & Herein & ESR herein & lQCD & Expt. \\
\hline$\pi$ & $\underline{0.138}$ & 0.138 & $/$ & $0.138(1)$ \\
$K$ & $\underline{0.495}$ & 0.416 & $/$ & $0.495(1)$ \\
$\eta_{c}$ & $\underline{\underline{2.984}}$ & 2.984 & $/$ & $2.984(1)$ \\
$\eta_{b}$ & $\underline{9.399}$ & 9.399 & $/$ & $9.399(1)$ \\
$B_{c}$ & 6.388 & 6.192 & $6.276(7)$ & $6.275(1)$ \\
$D$ & 1.771 & 1.561 & $1.868(3)$ & $1.868(1)$ \\
$D_{s}^{ \pm}$ & 1.981 & 1.839 & $1.968(4)$ & $1.968(1)$ \\
$B$ & 5.324 & 4.769 & $5.283(8)$ & $5.279(1)$ \\
$B_{s}^{ \pm}$ & 5.478 & 5.047 & $5.366(8)$ & $5.367(1)$ \\
\hline \hline
\end{tabular}


TABLE II. Masses of vector mesons calculated herein compared with those from the ESR herein, the LCD and the experiment. The 1QCD results are taken from: $M_{\rho}-$ Ref. [82]; $M_{K^{*}}$-Ref. [83]; $M_{\phi}$-Ref. [84]; $M_{J / \Psi}$-Ref. [85]; $M_{B_{c}}-$ Ref. [79]; $M_{D^{*}}, M_{D_{s}^{ \pm *}}, M_{B^{*}}$ and $M_{B_{s}^{ \pm *}}-$ Ref. [86]. The experimental data (labeled as Expt.) are taken from Ref. [1].

\begin{tabular}{lcclc}
\hline \hline Meson & Herein & ESR herein & \multicolumn{1}{c}{ lQCD } & Expt. \\
\hline$\rho$ & 0.749 & 0.749 & $0.780(16)$ & $0.775(1)$ \\
$K^{*}$ & 0.953 & 0.919 & $0.993(1)$ & $0.896(1)$ \\
$\phi$ & 1.089 & 1.089 & $1.032(16)$ & $1.019(1)$ \\
$J / \Psi$ & 3.122 & 3.122 & $3.098(3)$ & $3.097(1)$ \\
$\Upsilon$ & 9.497 & 9.497 & $/$ & $9.460(1)$ \\
$B_{c}^{*}$ & 6.542 & 6.310 & $6.331(7)$ & $/$ \\
$D^{*}$ & 1.988 & 1.936 & $2.013(14)$ & $2.009(1)$ \\
$D_{s}^{ \pm *}$ & 2.206 & 2.106 & $2.116(11)$ & $2.112(1)$ \\
$B^{*}$ & 5.501 & 5.123 & $5.321(8)$ & $5.325(1)$ \\
$B_{s}^{ \pm *}$ & 5.635 & 5.293 & $5.412(6)$ & $5.415(2)$ \\
\hline \hline
\end{tabular}

$$
m_{c}^{\zeta 19}=0.82 \mathrm{GeV}, \quad m_{b}^{\zeta 19}=3.59 \mathrm{GeV}
$$

we obtain the masses and leptonic decay constants of heavy quark mesons which are listed in Tables I and II. The renormalization-group-invariant masses are $\hat{m}_{c}=$ $1.61 \mathrm{GeV}, \hat{m}_{b}=7.16 \mathrm{GeV}$. The one-loop-evolved masses at $2 \mathrm{GeV}$ are $m_{c}^{2 \mathrm{GeV}}=1.22 \mathrm{GeV}, m_{b}^{2 \mathrm{GeV}}=5.41 \mathrm{GeV}$. The Euclidean constant quark masses are

$$
M_{c}^{E}=1.32 \mathrm{GeV}, \quad M_{b}^{E}=4.22 \mathrm{GeV} .
$$

\section{B. Results of heavy-light mesons}

With the parameters fixed in previous subsections, we can directly compute the properties of heavy-light mesons since the kernel beyond the ladder approximation, i.e., Eq. (28), does not introduce any new parameters. In this work, we mainly focus on the mass spectrum and the leptonic decay constants.

\section{Mass spectra}

We first compute the masses of the pseudoscalar and vector mesons. The obtained results and the comparisons with experimental data and lattice-QCD results are plotted in Figs. 4 and 5. The concrete data are summarized in Table I and Table II. Among them, $\pi, K, \eta_{c}, \eta_{b}$ are taken as the calibration to determine the parameters. Except for the heavy-light mesons, i.e., $D, D_{s}^{ \pm}, B$ and $B_{s}^{ \pm}$, the kernels used for others are actually the ladder approximation because the interactions of the two flavors are the same in Eq. (28).

Following Ref. [50], we also include the results given by the equal spacing rule (ESR). The key of the ESR is to define a passive-mass for the constituent-quark via

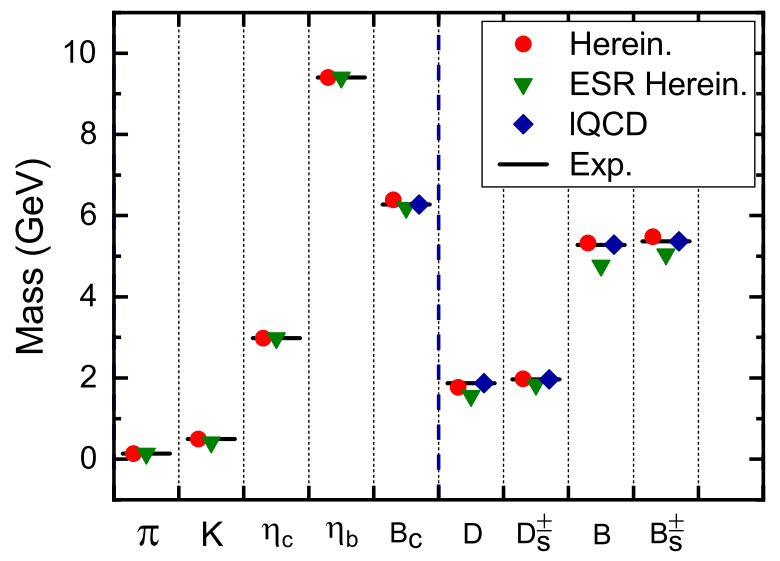

FIG. 4. Masses of pseudoscalar mesons calculated herein compared with those from the equal spacing rule herein (ESR herein), the lQCD and the experiments. The vertical blue dashed line is used to separate the light-light and heavy-heavy mesons (in the left region) with the heavy-light mesons (in the right region). The detailed numbers are listed in Table I.

$$
M_{X}^{f}=\frac{1}{2} m_{X_{f \bar{f}}},
$$

where $m_{X_{f \bar{f}}}$ denotes the mass of the $f$-flavor-symmetric meson in the channel $X$. The computed values (in $\mathrm{GeV}$ ) are:

$$
\text { PS: } \begin{array}{l|cccc}
f & u=d & s & c & b \\
\hline M_{X}^{f} & 0.07 & 0.35 & 1.49 & 4.70
\end{array},
$$

and

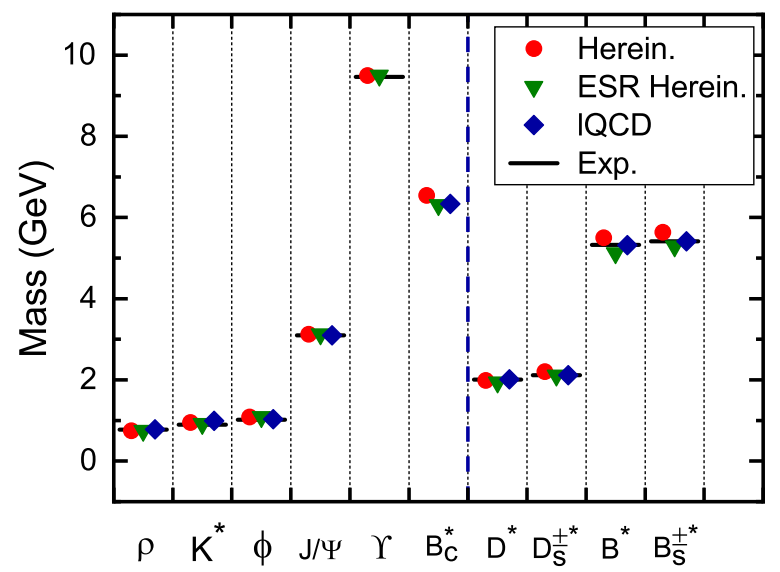

FIG. 5. Masses of vector mesons calculated herein compared with those from the ESR herein, the 1QCD and the experiments. The vertical blue dashed line is used to separate the light-light and heavy-heavy mesons (in the left region) with the heavy-light mesons (in the right region). The detailed numbers are listed in Table II. 


$$
\mathrm{VC}: \begin{array}{l|cccc}
f & u=d & s & c & b \\
\hline M_{X}^{f} & 0.37 & 0.54 & 1.56 & 4.75
\end{array}
$$

where PS, VC stands for the pseudoscalar, vector mesons, respectively. Then, the masses of flavor-unsymmetric mesons can be obtained by a straightforward interpolation as

$$
m_{X_{f \bar{g}}}=M_{X}^{f}+M_{X}^{g} .
$$

It is found that the masses of all pseudoscalar and vector mesons can be well reproduced with the BSE beyond the ladder approximation. The relative errors between the results herein and the experimental data are at the level of $5 \%$. For the $B_{c}^{*}$ meson, as the lack of the experimental data, we compare the result herein with the lattice-QCD and obtain a relative error only $4 \%$. On the other hand, the masses obtained with the ESR are comparable with the experimental data and the lattice-QCD, and the corresponding errors are just slightly larger than those with the BSE.

As mentioned above, the kernel, i.e., Eq. (28), actually expresses the averaging interaction of the light and heavy quarks. To demonstrate how the average works, we define the effective arithmetic average as

$$
K=-\gamma \otimes \gamma\left[\eta D^{q}+(1-\eta) D^{Q}\right],
$$

where $\eta \in[0,1]$ is the weight parameter. For $\eta=1$, the kernel is the same as the one for flavor-symmetric light quarks. In this case, the interaction strength of the kernel is quite large and the binding between the light and heavy quarks are too strong, accordingly. The resulting meson mass is smaller than the experimental data. On the other hand, for $\eta=0$, the kernel is the same as the one for flavorsymmetric heavy quarks. In this case, the interaction strength of the kernel is rather small and the binding between the light and heavy quarks are too weak, accordingly. The resulting meson mass is heavier than the experimental data.

Now we can take $D$ meson as an example to analyze the mass with the running of the weight parameter. The result is shown in Fig. 6. It is found that the weight parameter $\eta^{*}$ corresponding to the experimental mass indeed lies between the two limits, i.e., $0<\eta^{*}<1$. Thus, the kernel beyond the ladder approximation, i.e., Eq. (28), which produces the experimental mass, has the interaction strength equivalent to that weighted by $\eta^{*}$. However, Eq. (28) is inspired by the AV-WTI and has no additional parameters. In other words, it expresses an implicit and automatic average. Moreover, the well-produced full mass spectra suggest that this kind of average is generally appropriate.

\section{Leptonic decay constants}

We then compute the leptonic decay constants of the pseudoscalar and the vector mesons. The obtained results

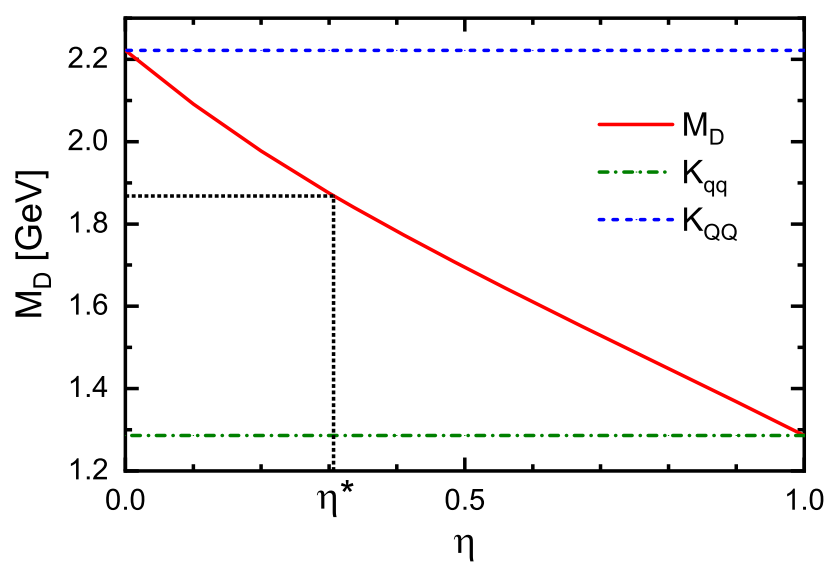

FIG. 6. Masses of $D$ meson with the weight parameter of the arithmetic average of the kernel, where $\eta^{*}$ denotes the value corresponding to the experimental mass.

are presented in Table III, Table IV, respectively. The comparisons with experimental data and lattice-QCD results are plotted in Figs. 7 and 8. Following Ref. [50], we also include the leptonic decay constants using the ESR, which is defined as

$$
f_{q Q} \approx f_{q Q}^{\text {interp }}:=\frac{1}{2}\left(f_{q q}+f_{Q Q}\right) .
$$

The first observation is that the leptonic decay constants are not as stable as the masses and the discrepancies from different sources become sizable for some mesons. For the pseudoscalar mesons of light, strange, and charm flavors (i.e., $\pi, K, \eta_{c}, D$, and $D_{s}^{ \pm}$), the theoretical methods produce similar values which are comparable with the experimental data. However the bottom-flavored pseudoscalar mesons (i.e., $\eta_{b}, B_{c}, B$, and $B_{s}^{ \pm}$) lack of experimental data and have very different theoretical results. The values produced with the BSE and the ESR are larger than lattice-QCD ones. Especially, for $B$ and $B_{s}^{ \pm}$, we have $f_{\mathrm{ESR}}>f_{\mathrm{BSE}}>f_{\mathrm{lQCD}}$.

TABLE III. Decay constants of pseudoscalar mesons calculated herein compared with those from the ESR, the IQCD and the experiments. The 1QCD results are taken from: $f_{\pi}$ and $f_{K}-$ Ref. [87]; $f_{\eta_{c}}, f_{\eta_{b}}$ and $f_{B_{c}}$ - Ref. [88]; $D, D_{s}^{ \pm}, B, B_{s}^{ \pm}-$Ref. [89]. The experimental data (labeled as Expt.) are taken from Refs. [1,90].

\begin{tabular}{lcccc}
\hline \hline Meson & Herein & ESR herein & lQCD & Expt. \\
\hline$\pi$ & 0.095 & 0.095 & $0.093(1)$ & $0.092(1)$ \\
$K$ & 0.113 & 0.115 & $0.111(1)$ & $0.110(1)$ \\
$\eta_{c}$ & 0.277 & 0.277 & $0.278(2)$ & $0.237(52)$ \\
$\eta_{b}$ & 0.559 & 0.559 & $0.472(5)$ & $/$ \\
$B_{c}$ & 0.429 & 0.418 & $0.307(10)$ & $/$ \\
$D$ & 0.169 & 0.186 & $0.150(1)$ & $0.144(4)$ \\
$D_{s}^{ \pm}$ & 0.212 & 0.206 & $0.177(1)$ & $0.182(3)$ \\
$B$ & 0.212 & 0.327 & $0.134(1)$ & $0.133(18)$ \\
$B_{s}^{ \pm}$ & 0.248 & 0.347 & $0.163(1)$ & $/$ \\
\hline \hline
\end{tabular}


TABLE IV. Decay constants of vector mesons calculated herein compared with those from the ESR, the lQCD and the experiments. The 1QCD results are taken from: $f_{\phi}$-Ref. [84]; $f_{J / \Psi}-$ Ref. [85]; $f_{\Upsilon}$-Ref. [91]; $f_{B_{c}^{*}}$-Ref. [92]; $f_{D^{*}}, f_{D_{s}^{ \pm *}}, f_{B^{*}}, f_{B_{s}^{ \pm *}}$-Ref. [86]. The experimental data (labeled as Expt.) are taken from Refs. [1,26].

\begin{tabular}{lcccc}
\hline \hline Meson & Herein & ESR herein & 1QCD & Expt. \\
\hline$\rho$ & 0.149 & 0.149 & $/$ & $0.153(1)$ \\
$K^{*}$ & 0.179 & 0.170 & $/$ & $0.159(1)$ \\
$\phi$ & 0.190 & 0.190 & $0.170(13)$ & $0.168(1)$ \\
$J / \Psi$ & 0.296 & 0.296 & $0.286(4)$ & $0.294(5)$ \\
$\Upsilon$ & 0.526 & 0.526 & $0.459(22)$ & $0.505(4)$ \\
$B_{c}^{*}$ & 0.483 & 0.411 & $0.298(9)$ & $/$ \\
$D^{*}$ & 0.199 & 0.222 & $0.158(6)$ & $/$ \\
$D_{s}^{ \pm *}$ & 0.256 & 0.243 & $0.190(5)$ & $/$ \\
$B^{*}$ & 0.246 & 0.338 & $0.131(5)$ & $/$ \\
$B_{s}^{ \pm *}$ & 0.283 & 0.358 & $0.158(4)$ & $/$ \\
\hline \hline
\end{tabular}

The reason why the ESR and the BSE produce large decay constants is that the interaction strength for the bottom sector is too strong since it is the same as that for the charm sector. This can be understood from the truth that $f_{\eta_{b}}$ obtained by the ESR and the BSE is significantly larger than that by lattice-QCD. If tuning $f_{\eta_{b}}$ down by decreasing the interaction strength for the bottom sector, the values of the BSE move close to the lattice-QCD results. However, the ESR still gives very large $f_{B}$ and $f_{B_{s}^{ \pm}}$. This means that the ESR always fails for extremely flavor-unsymmetric mesons.

For vector mesons, the situation is analogous and the differences between the BSE/ESR and lattice-QCD results are generally similar to those for pseudoscalar mesons. However, for the flavor-unsymmetric vector mesons, i.e., $D^{*}$ and $B^{*}$, the differences are significant. Note that $f_{\Upsilon}$ obtained by the BSE/ESR is larger than that by lattice-QCD

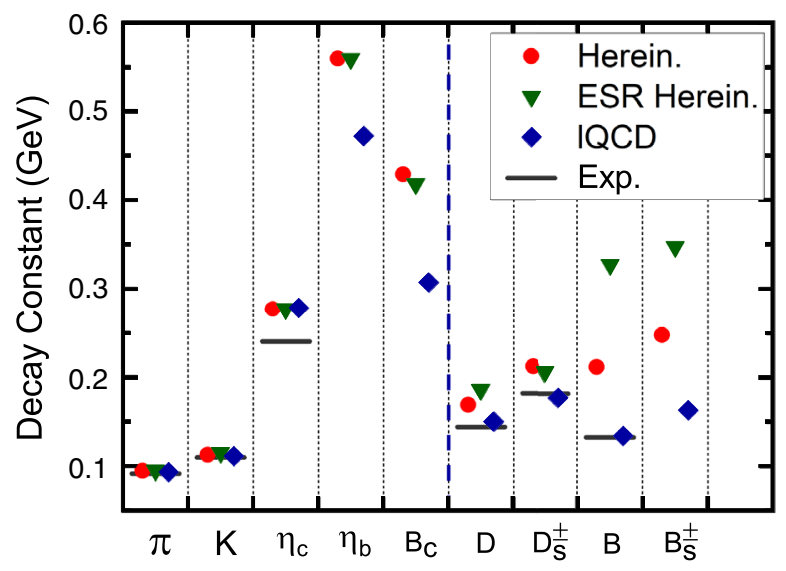

FIG. 7. Decay constants of pseudoscalar mesons calculated herein compared with those from the ESR herein, the IQCD and the experiments. The vertical blue dashed line is used to separate the light-light and heavy-heavy mesons (in the left region) with the heavy-light mesons (in the right region). The detailed numbers are listed in Table III.

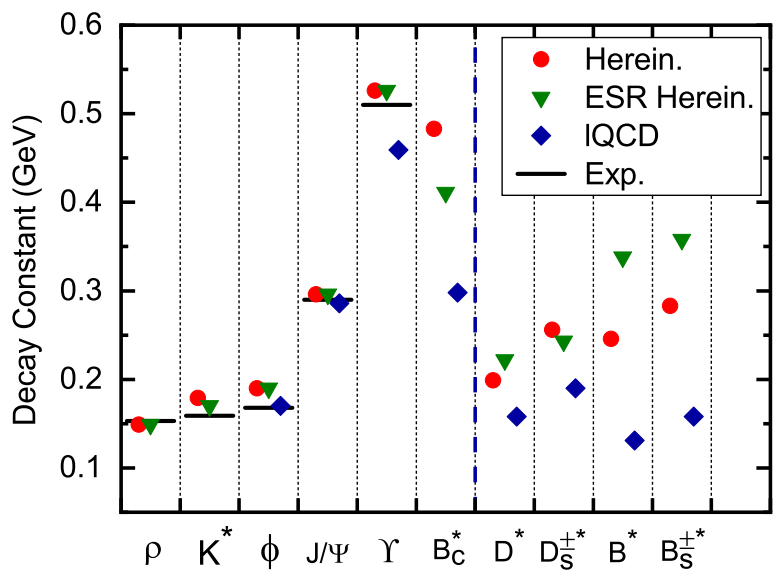

FIG. 8. Decay constants of vector mesons calculated herein compared with those from the ESR herein, the 1QCD and the experiments. The vertical blue dashed line is used to separate the light-light and heavy-heavy mesons (in the left region) with the heavy-light mesons (in the right region). The detailed numbers are listed in Table IV.

just as $f_{\eta_{b}}$. This is also a signature for that the interaction strength of the BSE/ESR is stronger than that of lattice QCD. If decreasing the interaction strength for the bottom sector as aforementioned, $f_{\Upsilon}$ decreases, and so does $f_{B^{*}}$. However, the differences between the BSE/ESR and latticeQCD results cannot be removed completely and remain noticeable. The reason is that the kernel in Eq. (28) only considers the AV-WTI which does not constrain the vector channel directly. Therefore, incorporating the axial-vector and vector WTIs to develop a universal BSE kernel for all channels could be the solution.

\section{SUMMARY}

In summary, the heavy-light mesons in pseudoscalar and vector channels are investigated in the framework of the Dyson-Schwinger equation approach of QCD. The rainbow-ladder truncation is modified under the consideration of the symmetries of QCD. It combines the effects of the light and heavy sectors. Moreover, the modified kernel involves no new parameter and can degenerate into the RL truncation for flavor-symmetric heavy-heavy or light-light mesons.

With the kernel beyond the ladder approximation, the calculated masses of the heavy-light mesons in pseudoscalar and vector channels agree with the experimental data at the level of 5\%. The masses obtained with the ESR are comparable with the experimental data and the latticeQCD results, while the corresponding errors are larger. From our analysis, the modified kernel expresses an implicit and automatic average with no additional averaging parameter. These facts shows that the modified kernel is appropriate to describe the mass spectrum of the ground states mesons. 
For the leptonic decay constants, our calculated results are not as stable as those for the masses. For the pseudoscalar mesons of light, strange and charm flavors, our theoretical methods produce similar values that are comparable with the experimental data. While for the bottom-flavored mesons, both the ESR and the BSE produce larger values than the lattice-QCD. The reason is that the interaction strength for the bottom sector is too strong since it is the same as that for the charm sector. For vector mesons, the differences between the BSE/ESR and lattice-QCD results are generally analogous but larger than those for pseudoscalar mesons. Besides the interaction strength, the missing constraint from the vector Ward-Takahashi identity is another factor to explain the larger differences.

From the discussions above, we can infer that the kernel beyond the ladder approximation is a promising tool to depict the quark-antiquark interactions. The mass spectra and the leptonic decay constants of the light-light, heavyheavy and heavy-light mesons in the pseudoscalar and vector channels can be well described, systematically. The modified kernel can be further improved by incorporating the axial-vector and vector Ward-Takahashi identities together and used to study meson and baryon properties. The related work is under progress.

\section{ACKNOWLEDGMENTS}

The authors are grateful for fruitful discussions with professor C. D. Roberts. The work was supported by the National Natural Science Foundation of China under Contracts No. 11435001, No. 11775041, No. 11847301, No. 11805024, and the National Key Basic Research Program of China under Contract No. 2015CB856900. This work was also supported in part by the Fundamental Research Funds for the Central Universities under Grant No. 2019CDJDWL0005.

\section{APPENDIX: DERIVATION OF THE KERNEL BEYOND LADDER APPROXIMATION}

The two-flavor AV-WTI, i.e., Eq. (23), reads

$$
\begin{gathered}
P_{\mu} \Gamma_{5 \mu}^{f g}(k, P)+i\left(m_{f}+m_{g}\right) \Gamma_{5}^{f g}(k, P) \\
=S_{f}^{-1}\left(k_{+}\right) i \gamma_{5}+i \gamma_{5} S_{g}^{-1}\left(k_{-}\right),
\end{gathered}
$$

where the vertices $\Gamma_{5 \mu}^{f g}$ and $\Gamma_{5}^{f g}$ satisfy the inhomogeneous Bethe-Salpeter equation (which is similar to Eq. (12) with inhomogeneous terms, $\gamma_{5} \gamma_{\mu}$ and $\gamma_{5}$, respectively) and the quark propagator $S_{f, g}$ satisfy the quark gap equation (with the Rainbow truncation $\left.\Gamma_{\nu}(q, P)=\gamma_{\nu}\right)$, i.e., Eqs. (1)-(2). Explicitly, we have:

$$
\begin{aligned}
\Gamma_{5 \mu}^{f g}(k, P) & =Z_{2} \gamma_{5} \gamma_{\mu}+\int_{q} K(q, k ; P) S_{f}\left(q_{+}\right) \Gamma_{5 \mu}^{f g}(q, P) S_{g}\left(q_{-}\right), \\
\Gamma_{5}^{f g}(k, P) & =Z_{2} Z_{m} \gamma_{5}+\int_{q} K(q, k ; P) S_{f}\left(q_{+}\right) \Gamma_{5}^{f g}(q, P) S_{g}\left(q_{-}\right), \\
S^{-1}(k) & =S_{0}^{-1}(k)+\int_{q} D_{\mu \nu}(q-k) \gamma_{\mu} S(q) \gamma_{\nu} .
\end{aligned}
$$

Inserting Eqs. (A2) into Eq. (A1) herein and noticing that $\left(k_{+}=k+\alpha P, k_{-}=k-(1-\alpha) P\right)$,

$$
\begin{gathered}
P_{\mu} Z_{2} \gamma_{5} \gamma_{\mu}+i\left(m_{f}+m_{g}\right) Z_{2} Z_{m} \gamma_{5} \\
=S_{0 f}^{-1}\left(k_{+}\right) i \gamma_{5}+i \gamma_{5} S_{0 g}^{-1}\left(k_{-}\right),
\end{gathered}
$$

we obtain the identity for the integral terms as

$$
\begin{aligned}
& P_{\mu} \int_{q} K(q, k ; P) S_{f}\left(q_{+}\right) \Gamma_{5 \mu}^{f g}(q, P) S_{g}\left(q_{-}\right) \\
& \quad+i\left(m_{f}+m_{g}\right) \int_{q} K(q, k ; P) S_{f}\left(q_{+}\right) \Gamma_{5}^{f g}(q, P) S_{g}\left(q_{-}\right) \\
& =\int_{q} D_{\mu \nu}(q-k) \gamma_{\mu} S_{f}\left(q_{+}\right) \gamma_{\nu} i \gamma_{5} \\
& \quad+i \gamma_{5} \int_{q} D_{\mu \nu}(q-k) \gamma_{\mu} S_{g}\left(q_{-}\right) \gamma_{\nu} .
\end{aligned}
$$

Rearranging the equation, one has

$$
\begin{array}{rl}
\int_{q} & K(q, k ; P) S_{f}\left(q_{+}\right)\left[P_{\mu} \Gamma_{5 \mu}^{f g}(q, P)\right. \\
& \left.+i\left(m_{f}+m_{g}\right) \Gamma_{5}^{f g}(q, P)\right] S_{g}\left(q_{-}\right) \\
& =-\int_{q} D_{\mu \nu}(q-k) \gamma_{\mu}\left[S_{f}\left(q_{+}\right) i \gamma_{5}+i \gamma_{5} S_{g}\left(q_{-}\right)\right] \gamma_{\nu} .
\end{array}
$$

Replacing the vertices in the left-hand side of the equation by the quark propagators (according to Eq. (A1) herein), one gets

$$
\begin{gathered}
\int_{q} K(q, k ; P) S_{f}\left(q_{+}\right)\left[S_{f}^{-1}\left(q_{+}\right) i \gamma_{5}+i \gamma_{5} S_{g}^{-1}\left(q_{-}\right)\right] S_{g}\left(q_{-}\right) \\
=-\int_{q} D_{\mu \nu}(q-k) \gamma_{\mu}\left[S_{f}\left(q_{+}\right) i \gamma_{5}+i \gamma_{5} S_{g}\left(q_{-}\right)\right] \gamma_{\nu} .
\end{gathered}
$$

Since the quark propagators with different flavors may have different effective interaction strengths, we take $D_{\mu \nu}^{f}, D_{\mu \nu}^{g}$ to specify each of them for clarity. One has, in turn, 


$$
\begin{aligned}
\int_{q} K(q, k ; P)\left[i \gamma_{5} S_{g}\left(q_{-}\right)+S_{f}\left(q_{+}\right) i \gamma_{5}\right] \\
=-\int_{q} \gamma_{\mu}\left[D_{\mu \nu}^{f}(q-k) S_{f}\left(q_{+}\right) i \gamma_{5}\right. \\
\left.\quad+D_{\mu \nu}^{g}(q-k) i \gamma_{5} S_{g}\left(q_{-}\right)\right] \gamma_{\nu} .
\end{aligned}
$$

This is exactly the appearance of Eq. (27).

As a special solution of the identity, one can equalize the terms inside the integrals on the both sides, i.e.,

$$
K\left(S_{f} \gamma_{5}+\gamma_{5} S_{g}\right)=-\gamma\left(D^{f} S_{f} \gamma_{5}+D^{g} \gamma_{5} S_{g}\right) \gamma,
$$

where the indices and momenta have been suppressed for simplicity. Accordingly, the kernel can be constructed as

$$
\begin{aligned}
& K_{1}=-\gamma \otimes\left(S_{f} \gamma_{5}+\gamma_{5} S_{g}\right)^{-1}\left(D^{f} S_{f} \gamma_{5}+D^{g} \gamma_{5} S_{g}\right) \gamma . \\
& K_{2}=-\gamma\left(D^{f} S_{f} \gamma_{5}+D^{g} \gamma_{5} S_{g}\right)\left(S_{f} \gamma_{5}+\gamma_{5} S_{g}\right)^{-1} \otimes \gamma .
\end{aligned}
$$

The kernel can also be constructed as

$$
K=\frac{1}{2} K_{1}+\frac{1}{2} K_{2}
$$

Note that the above kernel $K$ is not the unique solution, since Eq. (27) is a complicated matrix-equation. The strategy in our work is to make a choice as simple as possible and check its phenomenological performance.

However, because of the inverse term, the kernel cannot be used directly for practical calculations, since in the ultraviolet limit, i.e., $q \rightarrow \infty$, the kernel diverges as $\left(D^{f}-D^{g}\right) / 0$. In order to fix this issue, we shift $\gamma_{5}$ in Eq. (A9) and propose the kernel as (the color matrices are suppressed)

$$
K=-\frac{1}{2} \gamma_{\mu} \otimes \Sigma \cdot D_{\mu \nu}^{\Sigma} \cdot \gamma_{\nu}-\frac{1}{2} \gamma_{\nu} \cdot D_{\mu \nu}^{\Sigma} \cdot \Sigma \otimes \gamma_{\mu},
$$

with

$$
\begin{aligned}
D_{\mu \nu}^{\Sigma} & =\left[D_{\mu \nu}^{f}(l) S_{f}\left(q_{+}\right)+D_{\mu \nu}^{g}(l) S_{g}\left(q_{-}\right)\right] \gamma_{5}, \\
\Sigma & =\gamma_{5}\left[S_{f}\left(q_{+}\right)+S_{g}\left(q_{-}\right)\right]^{-1} .
\end{aligned}
$$

It is evident that Eq. (A10) is just the Eq. (28).

Since the $\Sigma$ and the $D_{\mu \nu}^{\Sigma}$ take the dressed effects into account, the kernel proposed in this work can be regarded as a model beyond the ladder approximation.
[1] M. Tanabashi et al. (Particle Data Group), Phys. Rev. D 98, 030001 (2018).

[2] E. Eichten and F. Feinberg, Phys. Rev. D 10, 3254 (1974).

[3] M. R. Pennington, J. Phys. Conf. Ser. 18, 1 (2005).

[4] A. Bashir, R. Bermudez, L. Chang, and C. D. Roberts, Phys. Rev. C 85, 045205 (2012).

[5] K. G. Wilson, Phys. Rev. D 10, 2445 (1974).

[6] P. H. Ginsparg and K. G. Wilson, Phys. Rev. D 25, 2649 (1982).

[7] G. G. Batrouni, G. R. Katz, A. S. Kronfeld, G. P. Lepage, B. Svetitsky, and K. G. Wilson, Phys. Rev. D 32, 2736 (1985).

[8] S. M. Ryan, Lect. Notes Phys. 889, 35 (2015).

[9] C. D. Roberts and A. G. Williams, Prog. Part. Nucl. Phys. 33, 477 (1994).

[10] C. D. Roberts and S. M. Schmidt, Prog. Part. Nucl. Phys. 45, S1 (2000).

[11] R. Alkofer and L. von Smekal, Phys. Rep. 353, 281 (2001).

[12] P. Maris and C. D. Roberts, Int. J. Mod. Phys. E 12, 297 (2003).

[13] C. D. Roberts, Prog. Part. Nucl. Phys. 61, 50 (2008).

[14] A. Bashir, L. Chang, I. C. Cloët, B. El-Bennich, Y. X. Liu, C. D. Roberts, and P. C. Tandy, Commun. Theor. Phys. 58, 79 (2012).

[15] I. C. Cloët and C. D. Roberts, Prog. Part. Nucl. Phys. 77, 1 (2014).

[16] C. Wetterich, Phys. Lett. B 301, 90 (1993).

[17] J. M. Pawlowski, Ann. Phys. (Amsterdam) 322, 2831 (2007).
[18] H. Gies, Lect. Notes Phys. 852, 287 (2012).

[19] E. E. Salpeter and H. A. Bethe, Phys. Rev. 84, 1232 (1951).

[20] N. Nakanishi, Prog. Theor. Phys. Suppl. 43, 1 (1969).

[21] H. J. Munczek, Phys. Rev. D 52, 4736 (1995).

[22] A. Bender, C. D. Roberts, and L. von Smekal, Phys. Lett. B 380, 7 (1996).

[23] P. Maris, C. D. Roberts, and P. C. Tandy, Phys. Lett. B 420, 267 (1998).

[24] M. R. Frank and C. D. Roberts, Phys. Rev. C 53, 390 (1996).

[25] P. Maris and C. D. Roberts, Phys. Rev. C 56, 3369 (1997).

[26] P. Maris and P. C. Tandy, Phys. Rev. C 60, 055214 (1999).

[27] M. S. Bhagwat, A. Holl, A. Krassnigg, C. D. Roberts, and P. C. Tandy, Phys. Rev. C 70, 035205 (2004).

[28] G. Eichmann, R. Alkofer, I. C. Cloet, A. Krassnigg, and C. D. Roberts, Phys. Rev. C 77, 042202(R) (2008).

[29] S. X. Qin, L. Chang, Y.X. Liu, C. D. Roberts, and D. J. Wilson, Phys. Rev. C 85, 035202 (2012).

[30] S. X. Qin, Few-Body Syst. 57, 1059 (2016).

[31] R. Casalbuoni, A. Beandrea, N. DiBartolomeo, R. Catto, F. Feruglio, and G. Nardulli, Phys. Rep. 281, 145 (1997).

[32] G. S. Bali, Phys. Rep. 343, 1 (2001).

[33] M. Blank and A. Krassnigg, Phys. Rev. D 84, 096014 (2011).

[34] T. Hilger, C. Popovici, M. Gómez-Rocha, and A. Krassnigg, Phys. Rev. D 91, 034013 (2015).

[35] C. S. Fischer, S. Kubrak, and R. Williams, Eur. Phys. J. A 51, 10 (2015). 
[36] M. H. Ding, F. Gao, L. Chang, Y. X. Liu, and C. D. Roberts, Eur. Phys. J. Web Conf. 113, 05020 (2016).

[37] J. Chen, M. H. Ding, L. Chang, and Y. X. Liu, Phys. Rev. D 95, 016010 (2017).

[38] M. A. Ivanov, Y. L. Kalinovsky, and C. D. Roberts, Phys. Rev. D 60, 034018 (1999).

[39] E. Rojas, B. El-Bennich, and J. P. B. C. de Melo, Phys. Rev. D 90, 074025 (2014).

[40] M. Gómez-Rocha, T. Hilger, and A. Krassnigg, Few-Body Syst. 56, 475 (2015).

[41] M. Gómez-Rocha, T. Hilger, and A. Krassnigg, Phys. Rev. D 92, 054030 (2015).

[42] M. Gómez-Rocha, T. Hilger, and A. Krassnigg, Phys. Rev. D 93, 074010 (2016).

[43] T. Hilger, M. Gómez-Rocha, A. Krassnigg, and W. Lucha, Eur. Phys. J. A 53, 213 (2017).

[44] M. A. Bedolla, E. Santopinto, and L. X. Gutirrez-Guerrero, Eur. Phys. J. Web Conf. 192, 00039 (2018).

[45] M. Y. Chen and L. Chang, Chin. Phys. C 43, 114103 (2019).

[46] D. Binosi, L. Chang, M. H. Ding, F. Gao, J. Papavassiliou, and C. D. Roberts, Phys. Lett. B 790, 257 (2019).

[47] P. L. Yin, C. Chen, G. Krein, C. D. Roberts, J. Segovia, and S. S. Xu, Phys. Rev. D 100, 034008 (2019).

[48] H. J. Munczek and A. M. Nemirovsky, Phys. Rev. D 28, 181 (1983).

[49] S. X. Qin, L. Chang, Y. X. Liu, C. D. Roberts, and D. J. Wilson, Phys. Rev. C 84, 042202(R) (2011).

[50] S. X. Qin, C. D. Roberts, and S. M. Schmidt, Phys. Rev. D 97, 114017 (2018).

[51] P. O. Bowman, U. M. Heller, D. B. Leinweber, M. B. Parappilly, and A. G. Williams, Phys. Rev. D 70, 034509 (2004).

[52] O. Oliveira and P. Bicudo, J. Phys. G 38, 045003 (2011).

[53] D. Binosi, L. Chang, J. Papavassiliou, and C. D. Roberts, Phys. Lett. B 742, 183 (2015).

[54] D. Binosi, C. Mezrag, J. Papavassiliou, C. D. Roberts, and J. Rodriguez-Quintero, Phys. Rev. D 96, 054026 (2017).

[55] J. Rodriguez-Quintero, D. Binosi, C. Mezrag, J. Papavassiliou, and C.D. Roberts, Few-Body Syst. 59, 121 (2018).

[56] C. Tang, F. Gao, and Y. X. Liu, Phys. Rev. D 100, 056001 (2019).

[57] D. Zwanziger, Phys. Rev. D 87, 085039 (2013).

[58] A. C. Aguilar, D. Binosi, J. Papavassiliou, and J. RodriguezQuintero, Phys. Rev. D 80, 085018 (2009).

[59] A. C. Aguilar, D. Binosi, and J. Papavassiliou, Phys. Rev. D 86, 014032 (2012).

[60] J. M. Cornwall, Phys. Rev. D 26, 1453 (1982).

[61] A. C. Aguilar, D. Ibanez, V. Mathieu, and J. Papavassiliou, Phys. Rev. D 85, 014018 (2012).

[62] X. Y. Xin, S. X. Qin, and Y. X. Liu, Phys. Rev. D 90, 076006 (2014).

[63] F. Gao, S. X. Qin, Y.X. Liu, C. D. Roberts, and S. M. Schmidt, Phys. Rev. D 89, 076009 (2014).
[64] F. F. Mojica, C. E. Vera, E. Rojas, and B. El-Bennich, Phys. Rev. D 96, 014012 (2017).

[65] M. Y. Chen, M. Ding, L. Chang, and C. D. Roberts, Phys. Rev. D 98, 091505 (2018).

[66] K. D. Bednar, I. C. Cloët, and P. C. Tandy, Phys. Lett. B 782, 675 (2018).

[67] S. X. Qin, C. D. Roberts, and S. M. Schmidt, Few-Body Syst. 60, 26 (2019).

[68] F. E. Serna, C. Chen, and B. El-Bennich, Phys. Rev. D 99, 094027 (2019).

[69] S.S. Xu, Z.F. Cui, L. Chang, J. Papavasilliou, C. D. Roberts, and H. S. Zong, Eur. Phys. J. A 55, 113 (2019).

[70] D. Binosi and R.-A. Tripolt, Phys. Lett. B 801, 135171 (2020).

[71] L. Chang, Y.X. Liu, M. S. Bhagwat, C. D. Roberts, and S. V. Wright, Phys. Rev. C 75, 015201 (2007).

[72] K. L. Wang, S. X. Qin, Y. X. Liu, L. Chang, C. D. Roberts, and S. M. Schmidt, Phys. Rev. D 86, 114001 (2012).

[73] C. H. Llewellyn-Smith, Ann. Phys. (N.Y.) 53, 521 (1969).

[74] A. Krassnigg, Phys. Rev. D 80, 114010 (2009).

[75] R. Williams, Eur. Phys. J. A 51, 57 (2015).

[76] N. Nakanishi, Phys. Rev. 138, B1182 (1965).

[77] N. Nakanishi, Phys. Rev. 139, B1401 (1965).

[78] S. X. Qin, C. D. Roberts, and S. M. Schmidt, Phys. Lett. B 733, 202 (2014).

[79] N. Mathur, M. Padmanath, and S. Mondal, Phys. Rev. Lett. 121, 202002 (2018).

[80] K. Cichy, M. Kalinowski, and M. Wagner, Phys. Rev. D 94, 094503 (2016).

[81] R. J. Dowdall, C. T. H. Davies, T. C. Hammant, and R. R. Horgan, Phys. Rev. D 86, 094510 (2012).

[82] Z. Fu and L. Wang, Phys. Rev. D 94, 034505 (2016).

[83] J. J. Dudek, R. G. Edwards, C. E. Thomas, and D. J. Wilson, Phys. Rev. Lett. 113, 182001 (2014).

[84] G. C. Donald, C. T. H. Davies, J. Koponen, and G. P. Lepage, Phys. Rev. D 90, 074506 (2014).

[85] G. C. Donald, C. T. H. Davies, R. J. Dowdall, E. Follana, K. Hornbostel, J. Koponen, G. P. Lepage, and C. McNeile, Phys. Rev. D 86, 094501 (2012).

[86] V. Lubicz, A. Melis, and S. Simula, Phys. Rev. D 96, 034524 (2017).

[87] E. Follana, C. T. H. Davies, G. P. Lepage, and J. Shigemitsu, Phys. Rev. Lett. 100, 062002 (2008).

[88] C. McNeile, C. T. H. Davies, E. Follana, K. Hornbostel, and G. P. Lepage, Phys. Rev. D 86, 074503 (2012).

[89] A. Bazavov et al. (for MILC Collaboration) Phys. Rev. D 98, 074512 (2018).

[90] K. W. Edwards et al. (CLEO Collaboration) Phys. Rev. Lett. 86, 30 (2001).

[91] B. Colquhoun, R. J. Dowdall, C. T. H. Davies, K. Hornbostel, and G. P. Lepage, Phys. Rev. D 91, 074514 (2015).

[92] B. Colquhoun, C. T. H. Davies, J. Kettle, J. Koponen, A. T. Lytle, R. J. Dowdall, and G. P. Lepage, Phys. Rev. D 91, 114509 (2015). 